\title{
PRIORIZAÇÃO DE STAKEHOLDERS: CONTRIBUIÇÃO DOS ESTUDOS TEÓRICOS E EMPÍRICOS
}

\section{STAKEHOLDER PRIORITIZATION: THEORETICAL AND EMPIRICAL CONTRIBUTIONS}

\author{
Data de submissão: 30/01/2015 \\ Aceite: $13 / 07 / 2015$ \\ Keysa Manuela Cunha De Mascena ${ }^{1}$ \\ JeongHeon $\mathrm{Kim}^{2}$ \\ Adalberto Américo Fischmann ${ }^{3}$ \\ Hamilton Luiz Corrêa ${ }^{4}$
}

\section{RESUMO}

A priorização de stakeholders é um tema ainda não consolidado na literatura, envolvendo diversos conceitos que não tornam claros quais os critérios que os gestores podem adotar para decidir quem será priorizado. O objetivo desta pesquisa, diante disso, é analisar os conceitos de priorização de stakeholders adotados na literatura. Para isso, realizou-se uma revisão teórica acerca da teoria dos stakeholders, da priorização de stakeholders e dos modelos de saliência e dominância de stakeholders, sendo coletados 214 artigos na base ISI Web of Knowledge, dos quais foram selecionados 55 artigos. Constatou-se que diferentes variáveis podem estar associadas à percepção de importância dos stakeholders, tais como a saliência, o poder, a legitimidade, a influência do stakeholder e a estratégia da empresa. Outras variáveis identificadas estão associadas ao atendimento dos interesses dos stakeholders, como a contribuição do stakeholder e a função objetivo da empresa. A contribuição desta pesquisa consiste, assim, em apresentar uma evolução do modelo de priorização de stakeholders baseado nos conceitos de percepção da importância dos stakeholders e no atendimento dos interesses dos stakeholders, propondo uma agenda de pesquisa para o tema.

Palavras-chave: Stakeholders. Priorização de stakeholders. Saliência de stakeholders.

\footnotetext{
${ }^{1}$ Possui graduação em Administração pela Universidade Federal do Ceará, mestrado em Administração pela Universidade de São Paulo. Atualmente é doutoranda em Administração pela Faculdade de Economia, Administração e Contabilidade da Universidade de São Paulo (FEAUSP). São Paulo. São Paulo. Brasil. E-mail: keysamascena@usp.br

${ }^{2}$ Possui graduação em Português e Estudo brasileiro pela Hankuk University of Foreign Studies e mestrado em andamento em Administração Geral pela Faculdade de Economia, Administração e Contabilidade - USP, FEAC/USP. São Paulo. São Paulo. Brasil. E-mail: j.h.kim@usp.br

${ }_{3}^{3}$ Possui graduação em Administração pela Universidade de São Paulo, USP e doutorado em Administração pela Universidade de São Paulo, USP. Atualmente é professor Titular da Faculdade de Economia, Administração e Contabilidade da Universidade de São Paulo (FEA-USP). São Paulo. São Paulo. Brasil. E-mail: aafischm@usp.br

${ }^{4}$ Possui graduação em Administração pela Universidade de São Paulo, USP, mestrado em Administração pela Universidade de São Paulo, USP e doutorado em Administração pela Universidade de São Paulo, USP. Atualmente é professor Doutor da FEA/USP, membro do Conselho de Graduação da USP, revisor de periódico da RAUSP - Revista de Administração e Membro de corpo editorial da Administrador Profissional. São Paulo. São Paulo. Brasil. E-mail: hamillco@usp.br
} 


\begin{abstract}
Stakeholder prioritization is a topic not yet consolidated in the literature, involving several concepts that do not make clear what criteria managers can take to decide who will be prioritized. The objective of this research is to analyze the stakeholder prioritization concepts adopted in the literature. The literature review is based on the stakeholder theory, stakeholder prioritization, stakeholder salience and dominance. We conducted a content analysis of fifty-five articles collected in ISI Web of Knowledge. It was noted that the different variables are likely to be associated with the perceived importance of stakeholder: salience, power, legitimacy, stakeholder influence and the company's strategy. Other variables are associated with satisfying the interests of stakeholders: the stakeholder contribution and the corporate objective function. The contribution of this research is to present an evolution of the stakeholder prioritization model based on the concepts regarding the perception of importance of stakeholders as well as based on satisfying the interests of stakeholders and propose a research agenda for the field.

Keywords: Stakeholders. Stakeholder prioritization. Stakeholder salience.
\end{abstract}

\title{
1 INTRODUÇÃO
}

A teoria dos stakeholders tem como marco a obra de Freeman (1984), Strategic Management: a stakeholder approach. Segundo a definição de Freeman (1984, p. 46), stakeholders são "grupos ou indivíduos que podem afetar ou são afetados pela organização na realização de seus objetivos".

A teoria dos stakeholders tem se desenvolvido nas últimas décadas, juntamente coma consideração dos interesses dos stakeholders que se disseminou nas organizações. Os interesses dos stakeholders passaram a ser levados em conta para o comportamento ético nos negócios e 0 atendimento às leis e normas socioambientais (MORAIS NETO; PEREIRA; MACCARI, 2012), bem como para as organizações responderem às pressões exercidas pelos stakeholders para que as corporações sejam cada vez mais sustentáveis (CARDOSO et al., 2008). O relacionamento e o diálogo com os stakeholders tornaram-se premissas para a sobrevivência das empresas, que passaram a incorporar valores que ultrapassam a visão estritamente econômica (TEIXEIRA; MORAES, 2013).

Nesse sentido, Evan e Freeman (1993) propuseram a função-objetivo da firma de servir como um veículo para coordenação dos interesses dos stakeholders. Esta função-objetivo da teoria dos stakeholders se contrapõe à função-objetivo da teoria da firma, que defende a maximização da riqueza dos acionistas. A função-objetivo da teoria dos stakeholders tornou-se objeto de crítica de alguns teóricos, que levantaram, segundo Phillips (2003b), interpretações equivocadas da teoria. Algumas destas interpretações são abordadas por Phillips (2003b) em sua discussão sobre os limites da teoria. Phillips (2003b), Phillips, Freeman e Wicks (2003) e Freeman et al. (2010) afirmam que alguns teóricos utilizaram interpretações equivocadas tanto para criticar a teoria dos stakeholders quanto para defendê-la. Uma das críticas apontadas consiste na afirmação de que todos os stakeholders devem ser tratados igualmente, conforme afirmam Gioia (1999), Marcoux (2000) e Sternberg (2000).

Este argumento encontra suporte na dimensão normativa da teoria, em que Donaldson e Preston (1995) defendem que todos os stakeholders têm interesses com valor intrínseco e não devem ser considerados apenas a partir de sua capacidade de promover os interesses de algum outro stakeholder. Por outro lado, na dimensão instrumental da teoria, Freeman (1999, p. 234) afirma que, "independente do objetivo da firma, a gestão eficiente deve gerenciar as relações que são importantes". Segundo Boaventura et al. (2013), esta afirmação sugere que alguns stakeholders podem ser considerados mais importantes, recebendo maior atenção por parte dos 
gestores. Nesse sentido, Phillips (2003b) acrescenta que a teoria dos stakeholders indica formas como os gestores podem priorizar os interesses de alguns stakeholders, utilizando, por exemplo, o critério de tratamento meritocrático e distribuição justa. Considera-se, portanto, que a priorização de stakeholders é uma questão inserida no debate teórico relacionado aos limites da teoria dos stakeholders e às dimensões da teoria.

Ao abordar a priorização de stakeholders, Phillips (2003b, p. 160) destaca que "uma questão que historicamente atormenta a teoria se refere a como os gestores alocam tempo, atenção, capacidade e outros recursos escassos entre os stakeholders". O autor acrescenta que a priorização pode ser interpretada de diferentes maneiras. Considerando que a priorização é tratada na literatura a partir de diferentes conceitos e modelos, o problema de pesquisa deste artigo consiste na falta de consolidação dos conceitos e modelos de priorização de stakeholders.

O objetivo geral desta pesquisa é, assim, analisar os conceitos de priorização de stakeholders adotados na literatura. Para atingir este objetivo, propõem-se os seguintes objetivos específicos:

a. descrever os conceitos, critérios ou modelos teóricos de priorização de stakeholders adotados na literatura;

b. analisar as contribuições das pesquisas sobre priorização de stakeholders.

A contribuição desta pesquisa consiste em apresentar uma consolidação dos estudos internacionais que abordam o conceito de priorização de stakeholders, evidenciando os avanços teóricos das pesquisas para o desenvolvimento de um modelo de priorização de stakeholders. Dessa forma, os achados podem auxiliar no desenvolvimento de futuras pesquisas sobre o tema.

\section{REFERENCIAL CONCEITUAL E TEÓRICO}

\subsection{Priorização de stakeholders}

O conceito de gestão de stakeholders proposto por Freeman (1984) trata do relacionamento da empresa com seus stakeholders. Para estabelecer este relacionamento, Freeman (1984) propõe um processo estratégico que inclui a identificação e análise de stakeholders, o desenvolvimento de estratégias e planos de ação e a implementação e o monitoramento dessas estratégias. Ao tratar da implementação estratégica, o conceito de priorização é discutido pelo autor, ainda que de forma implícita, ao afirmar que os recursos devem ser direcionados aos stakeholders mais importantes, ou seja, àqueles "que são vitais para o sucesso futuro da empresa" (FREEMAN, 1984, p. 159). Concluindo sua obra, o autor ainda discute a questão da primazia dos shareholders, owners ou acionistas, ao questionar se gestores deveriam atentar apenas para os interesses dos acionistas ou deveriam considerar os interesses dos demais stakeholders. Esta discussão é desenvolvida em obra posterior de Evan e Freeman (1993), que propõem que a função-objetivo da firma deveria ser a de servir como um veículo para coordenação dos interesses dos stakeholders. Ao empregar o termo coordenação, os autores não deixam claros critérios de priorização, mas rejeitam a primazia do acionista como proposto pela função-objetivo da teoria da firma.

Ao desenvolver o conceito de stakeholders, Clarkson (1995) sugere classificá-los como primários ou secundários. Os stakeholders primários são aqueles sem os quais a empresa não poderia sobreviver, havendo alto grau de interdependência entre a corporação e seus stakeholders primários. Para o autor, os stakeholders primários geralmente são: acionistas, investidores, empregados, clientes, fornecedores, governo e comunidade. Os stakeholders secundários são 
aqueles que influenciam ou afetam a organização, ou são influenciados ou afetados por ela, mas não possuem relação direta com a corporação. $O$ autor classifica a mídia e os grupos de interesse como stakeholders secundários, pois, apesar de não serem essenciais para a sobrevivência da organização, podem exercer influência na percepção que os stakeholders primários têm da organização. Segundo o argumento do autor, pode-se considerar que os stakeholders primários são mais importantes que os secundários.

Por outro lado, Donaldson e Preston (1995, p. 68) argumentam que, em uma dimensão normativa, "todas as pessoas ou grupos com interesses legítimos que participam de uma empresa buscam obter benefícios e que não haveria a priorização 'prima facie' de um conjunto de interesses e benefícios em detrimento de outro" (grifos do autor).

\subsubsection{Saliência de stakeholders}

Segundo Mitchell, Agle e Wood (1997, p. 854), a saliência dos stakeholders é definida como o "grau em que os gestores dão prioridade às reivindicações concorrentes dos stakeholders". Segundo os autores, os stakeholders podem ser identificados a partir de três atributos: (1) o poder dos stakeholders de influenciar a organização; (2) a legitimidade da relação do stakeholder com a organização; e (3) a urgência das reivindicações do stakeholder na organização. Dessa forma, a saliência do stakeholder está positivamente relacionada com a percepção gerencial da presença desses três atributos.

Poder é considerado pelos autores como a influência de um ator social sobre outro para que ele faça algo que não faria sem essa influência. O poder pode ser coercitivo, utilitário ou normativo (ETZIONI, 1964). Legitimidade refere-se à adequação das ações de um stakeholder às normas, aos valores e às crenças socialmente aceitas (SUCHMAN, 1995), podendo ser considerada nos níveis individual, organizacional e social (WOOD, 1991). Urgência diz respeito ao grau de atenção imediata às reivindicações do stakeholder, que está relacionado ao tempo e à importância da reivindicação ou da relação com o stakeholder (MITCHELL; AGLE; WOOD, 1997).

Mitchell, Agle e Wood (1997) desenvolveram uma classificação a partir desses três atributos. Inicialmente, os stakeholders que possuem apenas um atributo são chamados de stakeholders latentes; aqueles que possuem dois atributos são os stakeholders expectantes; e os que possuem os três atributos são os stakeholders definitivos. Essa tipologia é apresentada na Figura 1.

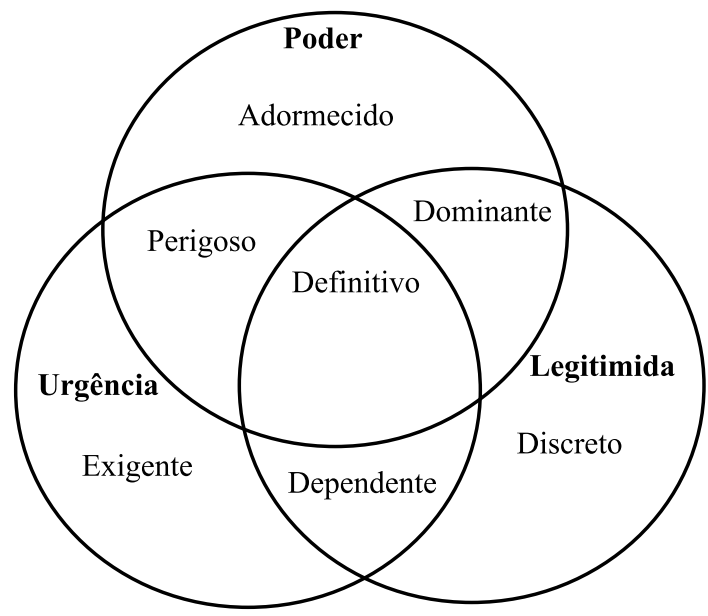

Figura 1 - Tipologia dos stakeholders

Fonte: Mitchell, Agle e Wood (1997, p. 874).

Os stakeholders latentes podem ser: (a) adormecidos - possuem um único atributo, o 
poder, mas, por não possuírem os outros, não o utilizam (os gestores devem estar atentos, pois esses stakeholders podem adquirir outro atributo); (b) discretos - possuem um único atributo, legitimidade, mas não tem poder nem urgência em suas reivindicações (os gestores podem direcionar ações de filantropia); (c) exigentes - possuem unicamente o atributo da urgência (podem ser protestantes insatisfeitos com a corporação, mas somente merecerão maior atenção se adquirirem mais um atributo).

Já os stakeholders expectantes podem ser: (a) dominantes - têm poder e legitimidade (as empresas devem buscar direcionar uma atenção especial aos interesses desses stakeholders e elaborar relatórios de prestação de contas para eles); (b) dependentes - possuem legitimidade e urgência (são assim chamados, pois dependem de outro stakeholder, que tenha poder, para influenciar a empresa para o atendimento de sua reivindicação); (c) perigosos - possuem urgência e poder (podem ser coercitivos e violentos).

Os stakeholders definitivos, por sua vez, possuem os três atributos: poder, legitimidade e urgência. Segundo o modelo, os gestores devem atender e priorizar os interesses desse tipo de stakeholder.

\subsubsection{Dominância de stakeholders}

A dominância de stakeholders refere-se à distribuição de benefícios realizada pela empresa entre seus stakeholders. Para fins operacionais, dominância é definida como a habilidade de determinado stakeholders obter recursos de forma preferencial em relação a outros stakeholders (BOAVENTURA et al., 2013).

A dominância difere do conceito de saliência de stakeholders proposto por Mitchell, Agle e Wood (1997). O conceito de saliência está ligado à percepção dos gestores sobre a importância dos stakeholders. O modelo de dominância mostra quais são, de fato, os níveis de benefícios recebidos pelos stakeholders. Em outras palavras, saliência é o que deve ser considerado pela gestão de stakeholders, e dominância é o resultado dessa gestão.

Com relação à priorização, pode-se afirmar que este conceito é discutido na literatura sob estas duas perspectivas: a percepção do gestor acerca da importância do stakeholder; e o atendimento dos interesses dos stakeholders. Essas perspectivas podem ser compreendidas por meio do modelo conceitual de Boaventura et al. (2013), apresentado na Figura 2.

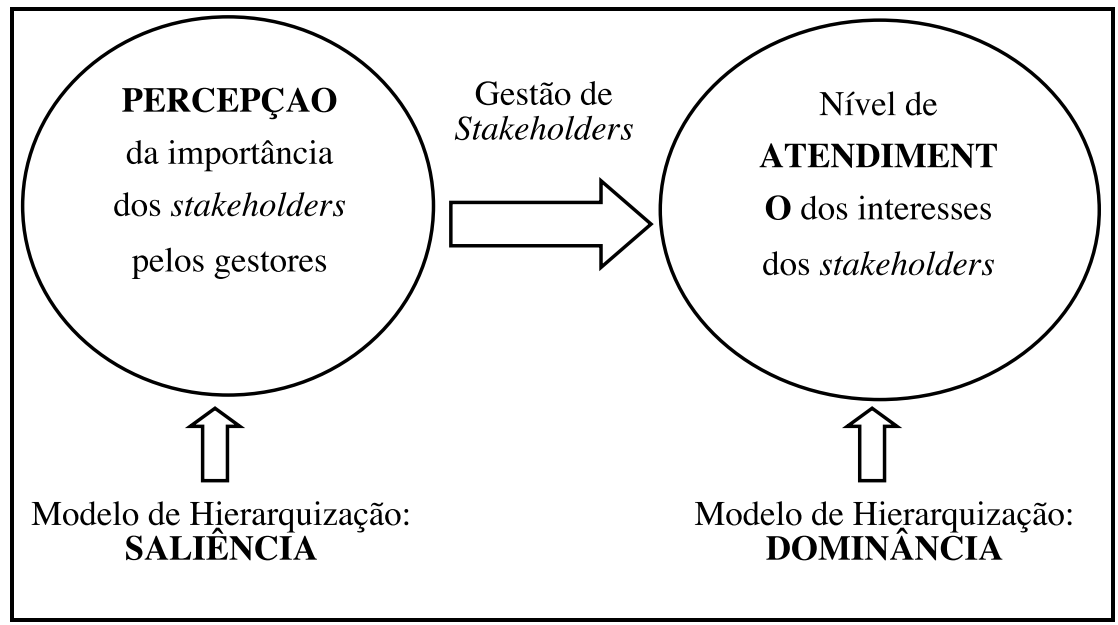

Figura 2 - Priorização de Stakeholders Fonte: Boaventura et al. (2013). 
A Figura 2 apresenta as duas formas de compreender a priorização de stakeholders na literatura e os conceitos relacionados. A primeira refere-se à percepção da importância dos stakeholders pelos gestores: os stakeholders priorizados são aqueles considerados mais importantes e que recebem maior atenção da gestão. Nesta perspectiva, uma forma de hierarquizar os stakeholders consiste no modelo de saliência de stakeholders. A segunda perspectiva refere-se ao nível de atendimento dos interesses dos stakeholders: os stakeholders priorizados são aqueles que recebem mais recursos do que os outros. Nesta perspectiva, uma forma de hierarquizar os stakeholders é a dominância de stakeholders.

Acrescenta-se que a gestão de stakeholders busca estabelecer uma conexão entre a percepção do gestor acerca da importância dos stakeholders e a maneira como os recursos organizacionais serão distribuídos entre eles. Em outras palavras, a gestão almeja alocar os recursos de forma eficiente, para que as demandas dos stakeholders mais importantes sejam atendidas de forma prioritária. Esta definição está relacionada ao conceito de Freeman (1999) de que a gestão eficiente deve gerenciar as relações que são importantes.

Este artigo considera o modelo proposto por Boaventura et al. (2013), apresentado na Figura 2, como o modelo conceitual para a análise da priorização de stakeholders. Portanto, acredita-se que a priorização de stakeholders pode ser tanto a percepção do gestor em relação à importância do stakeholder quanto o nível de atendimento de interesses recebido pelo stakeholder.

\section{METODOLOGIA}

Empreendeu-se uma pesquisa descritiva, levantando-se as características da produção científica sobre priorização de stakeholders (HAIR Jr. et al., 2005). A pesquisa também é bibliométrica, pois descreve as características de artigos científicos publicados (ARAÚJO, 2006).

\subsection{Coleta de dados}

A coleta de dados foi realizada nos meses de abril e maio de 2014 na base de dados ISI Web of Knowledge. Nos critérios de busca, foram delimitados a área (business economics) e o tipo de documento (article). As palavras-chave utilizadas para busca foram a combinação da palavra stakeholder no título com as palavras prioritization, priority, salience, importance, primacy, preference, hierarchy, ranking, dominance e superiority no título, no resumo ou nas palavras-chave.

\subsection{Análise de conteúdo}

Os dados foram analisados utilizando-se a técnica de análise categorial, uma das técnicas de análise de conteúdo (BARDIN, 1977). Esta pesquisa associa o conteúdo dos artigos a categorias, utilizando-se, então, de análise de conteúdo. As categorias definidas para análise descritiva dos artigos têm como objetivo caracterizar a amostra de acordo com os temas e métodos de pesquisa adotados.

\subsubsection{Análise descritiva dos artigos}

$\mathrm{Na}$ análise descritiva, os artigos foram classificados nas categorias: a) periódico; b) abordagem metodológica; e c) delineamento da pesquisa. Para a categoria abordagem metodológica, foram consideradas as subcategorias elencadas por Machado-da-Silva, Amboni e Cunha (1989, p. 1604): 
Empírica: concentra-se na observação e na análise dos dados, sem relacioná-los a um quadro referencial teórico específico para a explicação das situações reais;

Teórico-empírica: estudos que partem de um quadro de referência teórico tentando, através da coleta e análise de dados, refutá-lo ou corroborá-lo no todo ou em parte;

Teórica: a análise atém-se a conceitos, proposições, identificação de variáveis ou construção/reconstrução de modelos sem implicar em teste empírico para corroborar ou refutar a teoria exposta ou, alternativamente, ensaio sobre determinado assunto podendo alcançar diferentes graus de profundidade.

Para a categoria delineamento da pesquisa, considerou-se a classificação de Gil (2008): estudo de caso, estudo de campo, survey e pesquisa documental.

\subsubsection{Análise teórica dos artigos}

$\mathrm{Na}$ análise teórica, os artigos foram classificados nas categorias: a) conceito - percepção ou atendimento; e b) critério ou modelo teórico de priorização.

As categorias de análise dos artigos baseiam-se no modelo proposto por Boaventura et al. (2013), que classifica a priorização como percepção da importância do stakeholder e como nível de atendimento dos interesses do stakeholder.

\section{APRESENTAÇÃO E ANÁLISE DOS DADOS}

A pesquisa na base de dados ISI Web of Knowledge, de acordo com os critérios detalhados na seção 3.1, permitiu localizar 214 artigos. Após retirar os artigos em duplicidade na busca, selecionaram-se 186 artigos. Destes não foi possível localizar, na íntegra, seis artigos. Portanto, a amostra selecionada para leitura prévia foi composta de 180 artigos. A partir da leitura dos artigos, selecionaram-se 55 artigos alinhados ao tema de pesquisa. Para considerar o artigo alinhado ao tema, o critério empregado foi o de avaliar se o artigo discutia sobre algum tipo de classificação hierárquica de stakeholders.

A quantidade de artigos publicada por ano é apresentada na Figura 3.

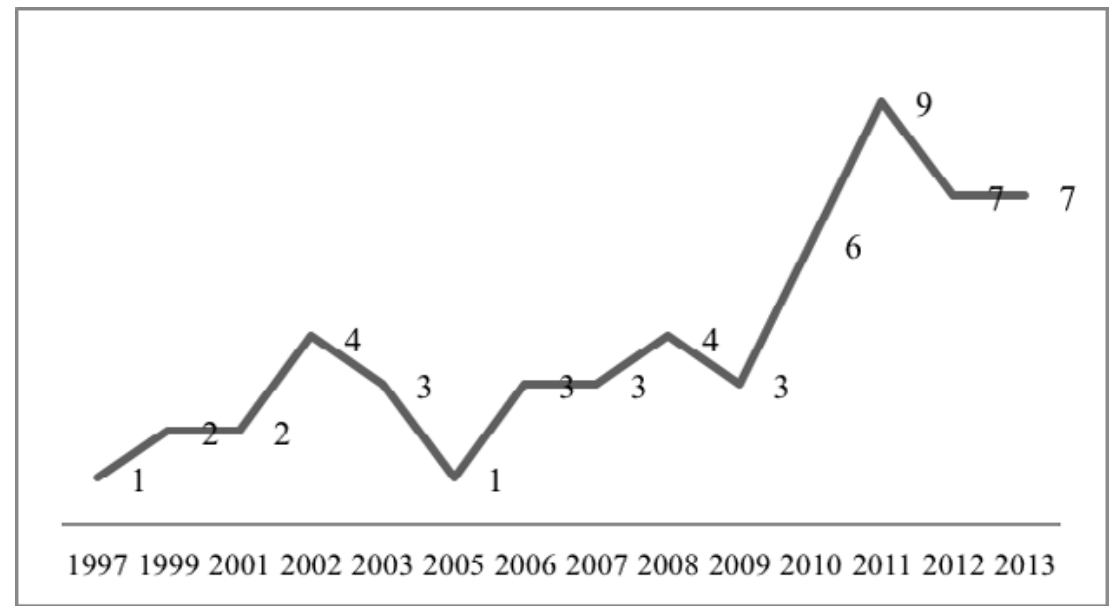

Figura 3 - Total de artigos por ano

Fonte: dados da pesquisa. 
Embora a coleta de artigos não tenha condicionado o filtro de ano da publicação, o primeiro ano em que foram encontrados artigos tratando do tema de priorização é 1997 , revelando que este tema é recente. A primeira publicação selecionada foi a de Mitchell, Agle e Wood (1997): trata-se de artigo com a proposta do modelo de saliência. No período de 1997 a 2009, foram selecionados 26 artigos, enquanto que, nos últimos três anos, período de 2010 a 2013, foram analisados 29 artigos abordando o tema de priorização.

\subsection{Análise descritiva}

A primeira análise foi a dos periódicos que possuíam maior volume de publicações na amostra de 55 artigos. Os seis periódicos que possuíam mais de uma publicação, ordenados de forma decrescente, são apresentados na Tabela 1.

Tabela 1 - Periódicos com mais publicações

\begin{tabular}{|c|c|}
\hline Periódico & Qtde \\
\hline Journal of Business Ethics & 11 \\
\hline Strategic Management Journal & 5 \\
\hline Academy of Management Review & 4 \\
\hline Business Ethics Quarterly & 4 \\
\hline Inzinerine Ekonomika-Engineering Economics & 2 \\
\hline Academy of Management Journal & 2 \\
\hline
\end{tabular}

Observa-se que o periódico com maior volume de publicações é o Journal of Business Ethics. Verificam-se, também, outros periódicos de alto impacto com volume de publicações no tema, como Strategic Management Journal, Academy of Management Review e Business Ethics Quarterly.

Os artigos foram classificados, ainda, de acordo com a abordagem metodológica. Encontraram-se 36 artigos teórico-empíricos, 15 artigos teóricos e quatro artigos empíricos.

Os artigos teórico-empíricos e empíricos foram classificados de acordo com o delineamento da pesquisa em estudo de caso, estudo de campo, survey e pesquisa documental, conforme apresentado na Tabela 2.

Tabela 2 - Delineamento da pesquisa

\begin{tabular}{c|c}
\hline Delineamento & Qtde \\
\hline Survey & 20 \\
\hline Estudo de caso & 10 \\
\hline Estudo de campo & 6 \\
\hline Pesquisa documental & 4 \\
\hline Total de artigos empíricos & $\mathbf{4 0}$ \\
\hline \multicolumn{2}{c}{ Fonte: dados da pesquisa. }
\end{tabular}

Embora o método predominante seja a survey, observa-se que muitos estudos empregam o método de estudo de caso, o que pode sugerir que os estudos de priorização adotam métodos mais exploratórios e qualitativos, como o de estudo de caso, bem como métodos mais descritivos e quantitativos, como a survey. 


\subsection{Análise teórica}

Classificando-se os artigos conforme as categorias propostas por Boaventura et al. (2013), de percepção da importância do stakeholder e atendimento dos interesses dos stakeholders, obtiveram-se 48 estudos sobre percepção, cinco estudos sobre atendimento e dois estudos que contemplam as duas dimensões - percepção e atendimento. Esta classificação teórica também pode ser considerada por suas características descritivas, conforme Tabela 3 e Figura 4.

Tabela 3 - Conceito de priorização e abordagem metodológica

\begin{tabular}{c|c}
\hline Conceito e abordagem metodológica & Qtde \\
\hline Percepção & $\mathbf{4 8}$ \\
\hline Empírica & 3 \\
\hline Teórica & 13 \\
\hline Teórico-empírica & 32 \\
\hline Atendimento & $\mathbf{5}$ \\
\hline Empírico & 1 \\
\hline Teórico & 1 \\
\hline Teórico-empírico & 3 \\
\hline Percepção \& Atendimento & $\mathbf{2}$ \\
\hline Teórico & 1 \\
\hline Teórico-empírico & 1 \\
\hline Total & $\mathbf{5 5}$ \\
\hline \multicolumn{2}{|c|}{ Fonte: dados da pesquisa. }
\end{tabular}

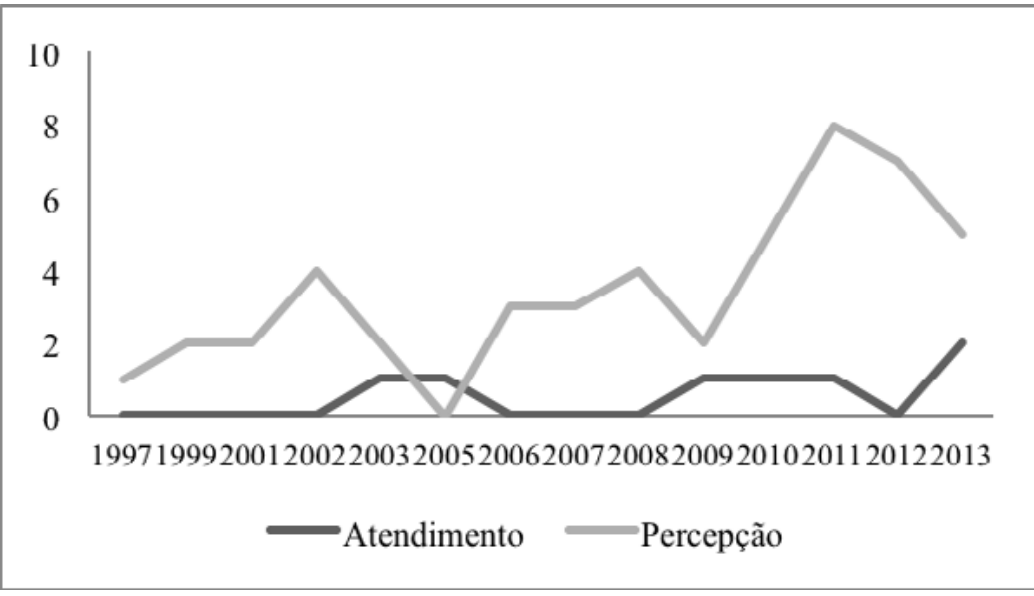

Figura 4 - Conceito de priorização por ano Fonte: dados da pesquisa.

Na Figura 4, consideraram-se os 48 estudos sobre percepção em comparação com os cinco estudos sobre atendimento, somados com os dois estudos que contemplam conjuntamente a percepção e o atendimento. Os dados mostram que a perspectiva de percepção da importância do stakeholder é amplamente estudada, não apresentando tendência de declínio no período, considerando-se as limitações da coleta de dados.

Os artigos foram analisados a fim de identificar os critérios ou modelos de priorização de stakeholders, categorizados na Tabela 4. 
Tabela 4 - Critérios ou modelos adotados pelos artigos

\begin{tabular}{c|c}
\hline Critérios ou modelos & Qtde \\
\hline Percepção & $\mathbf{4 8}$ \\
\hline Saliência de stakeholders & 36 \\
\hline Importância do stakeholder & 3 \\
\hline Orientação para stakeholders & 3 \\
\hline Estratégias ambientais & 2 \\
\hline Influência dos stakeholders & 2 \\
\hline Pressão dos stakeholders & 1 \\
\hline Modelo INSPIRE & 1 \\
\hline Atendimento & $\mathbf{5}$ \\
\hline Justiça/fairness & 1 \\
\hline Contribuição do stakeholder & 1 \\
\hline Stakeholder integration capability & 1 \\
\hline Engajamento em programas de RSC & 1 \\
\hline Hierarquização da função-objetivo & 1 \\
\hline Percepção \& Atendimento & $\mathbf{2}$ \\
\hline Pressão dos stakeholders & 1 \\
\hline Fairness/legitimidade & 1 \\
\hline Total & $\mathbf{5 5}$ \\
\hline Fonte: dados da pesquisa. &
\end{tabular}

Observa-se uma diversidade de critérios e modelos adotados nos estudos de priorização. Porém, pode-se destacar o modelo de saliência de stakeholders de Mitchell, Agle e Wood (1997) como o mais utilizado, com 36 artigos analisados. Os achados evidenciam a aceitação do modelo de saliência como um modelo de priorização e hierarquização de stakeholders. As subseções a seguir tratam de cada um dos temas apresentados na Tabela 4.

\subsubsection{Percepção da importância dos stakeholders}

Os artigos que abordam a priorização de stakeholders relacionada ao conceito da percepção da importância dos stakeholders adotam diferentes critérios de priorização que foram agrupados em sete temas: saliência de stakeholders, importância do stakeholder, orientação para stakeholders, estratégias ambientais, pressão dos stakeholders, influência dos stakeholders e modelo INSPIRE.

Saliência de stakeholders: o modelo de saliência de Mitchell, Agle e Wood (1997) é um importante modelo de priorização de stakeholders, conforme tratado na seção 2.2.1. A análise das publicações revelou que 33 artigos abordam o tema de saliência, incluindo o referido artigo de proposição do modelo. Dentre os artigos teóricos mais recentes, destacam-se os que procuram contribuir para o modelo, como o de Neville, Bell e Whitwell (2011), que argumentam que a urgência não é um atributo importante, que a legitimidade moral da reivindicação é um atributo principal e que a saliência varia em função dos diferentes graus dos atributos de poder, legitimidade e urgência. Além disso, Tashman e Raelin (2013) argumentam que a percepção dos gestores da saliência dos stakeholders pode ser viesada em função da racionalidade limitada, da assimetria de informações, do comportamento oportunista e de interesses conflitantes de stakeholders. Para minimizar este viés, os autores sugerem o conceito de stakeholder salience to the firm como o grau em que os gestores devem identificar e gerenciar as reivindicações dos stakeholders. 
Importância do stakeholder: Guild (2002) evidencia a importância dos stakeholders revelada nos discursos dos gestores e no diálogo com os stakeholders. Jepsen e Eskerod (2009) debatem a importância de classificar os stakeholders pela importância percebida pelos gestores na análise de stakeholders em projetos. Segui-Alcaraz (2012) busca classificar organizações de crédito segundo o grau de importância que é atribuído aos stakeholders em iniciativas de responsabilidade social. Esses três estudos abordam a perspectiva da percepção dos gestores quanto à importância dos stakeholders.

Orientação para stakeholders: os artigos classificados neste tema destacam a percepção de importância de um stakeholder em relação a outros. Adams, Licht e Sagiv (2011) e Neumann, Roberts e Cauvin (2011) tratam da importância dos shareholders em relação aos demais stakeholders e De Bussy e Suprawan (2012) discorrem acerca da importância dos funcionários em relação a outros stakeholders primários: shareholders, clientes, fornecedores e comunidade.

Estratégias ambientais: a priorização também é estudada com relação às estratégias ambientais da empresa. Henriques e Sadorsky (1999) classificam as empresas estudadas conforme o tipo de estratégia ambiental, mencionando que, de acordo com o tipo de estratégia adotada, há diferenças na percepção de quais são os stakeholders mais importantes. Buysse e Verbeke (2003) também analisam que tipo de estratégia ambiental está associado à gestão e priorização de stakeholders.

Pressão dos stakeholders: Murillo-Luna, Garces-Ayerbe e Rivera-Torres (2008) analisam as pressões exercidas por diferentes grupos de stakeholders para que as empresas adotem medidas de proteção ao meio ambiente. Os autores investigam se os gestores percebem algum grupo de stakeholder mais importante e como os stakeholders influenciam a gestão ambiental da empresa.

Influência dos stakeholders: Pedersen (2011) investiga o tema de priorização, questionando os gestores sobre o quanto os stakeholders influenciam ou afetam a empresa. Em seus resultados, contata que aqueles considerados com maior influência na amostra estudada, funcionários e clientes, são os mais priorizados. Chigona et al. (2010) também consideram a percepção de importância dos stakeholders e sua capacidade de influência, classificando os stakeholders de acordo com um mapa de importância e influência.

Modelo INSPIRE: Frooman (2010) propõe um modelo de gestão de stakeholders com cinco dimensões: issues identification, network formulation, stakeholder prioritization, intervention analysis and response engagement. Na dimensão de priorização de stakeholders, é considerada a saliência ou o poder e interesse dos stakeholders, bem como o ciclo de vida e sistema de incentivos dos gestores da organização.

\subsubsection{Atendimento dos interesses dos stakeholders}

Os artigos que abordam a priorização de stakeholders relacionada ao conceito de atendimento dos interesses dos stakeholders também adotam diferentes critérios de priorização que foram agrupados em cinco temas: justiça/fairness, contribuição do stakeholder, stakeholder integration capability, hierarquização da função-objetivo e engajamento em programas de Responsabilidade Social Corporativa (RSC).

Justiça/fairness: Aguiar, Becker e Miller (2013) tratam da justiça distributiva na ótica de imparcialidade, que exige dar atenção igual ou pelo menos adequada aos interesses de todos os stakeholders. Ao apontar que diferentes teorias da justiça distributiva resolveram a questão da imparcialidade de forma diferente, eles analisaram três mecanismos diferentes para atingir a imparcialidade em matéria de justiça distributiva. 
Contribuição do stakeholder: segundo Cameron et al. (2011), priorização de stakeholders é interpretada como definir os pesos para a importância de diferentes stakeholders. Os autores ressaltam que os stakeholders devem ser priorizados por suas contribuições e definiram o conceito de contribuição como o valor que os stakeholders oferecem para a empresa. Com base nesta conceituação, um método é proposto para determinar os pesos relativos, com o objetivo final de determinar a alocação adequada dos recursos da empresa.

Stakeholder integration capability: Driessen e Hillebrand (2013) conceituam capacidade de integração dos stakeholders como a combinação de técnicas de identificação de stakeholders, mecanismos de coordenação e princípios de priorização. Os autores propõem que a identificação de stakeholder issues leva as organizações a desenvolverem mecanismos de coordenação e princípios de priorização. Segundo os autores, é importante fazer a distinção entre stakeholders de mercado e de não mercado, embora nem todas as organizações sejam capazes de identificar os issues que são importantes para ambos os stakeholders.

Hierarquização da função-objetivo: Boaventura et al. (2009) verificaram se há ou não subordinação da função-objetivo dos stakeholders à dos shareholders. Para operacionalizar a pesquisa, os autores investigaram se os stakeholders eram atendidos caso anteriormente os shareholders não tenham sido atendidos. Os autores verificaram que não há subordinação da função -objetivo da teoria dos stakeholders na amostra estudada, ou seja, os demais stakeholders foram atendidos mesmo quando os shareholders não foram.

Engajamento em programas de RSC: Knox, Maklan e French (2005) definiram engajamento de stakeholders como identificação e priorização dos stakeholders, comunicação com os stakeholders e conexão dos programas de RSC com os negócios e resultados sociais. Como o impacto positivo da RSC sobre os principais stakeholders da empresa foi verificado empiricamente, o engajamento de stakeholders foi considerado uma importante estratégia.

\subsubsection{Percepção e atendimento}

Os artigos que abordam a priorização de stakeholders tanto na perspectiva da percepção da importância quanto na perspectiva do atendimento dos interesses dos stakeholders foram agrupados em dois temas: pressão dos stakeholders e fairness/legitimidade.

Pressão dos stakeholders: Vazquez-Brust et al. (2010) trataram do atendimento às pressões dos stakeholders para proteção do meio ambiente, analisando se o atendimento da empresa à pressão que o stakeholder exerce está relacionado com a saliência do stakeholder percebida pelos gestores. Os autores verificaram que existem diferenças entre a percepção do gestor acerca da importância do stakeholder e a forma como o stakeholder é tratado pela gestão.

Fairness/legitimidade: Phillips (2003a), em seu artigo teórico intitulado Stakeholder Legitimacy, aborda conceitos relacionados tanto à percepção da importância quanto ao atendimento de interesses dos stakeholders. O conceito de percepção da importância está relacionado com a definição de legitimidade normativa e derivativa. Os stakeholders com legitimidade normativa são considerados mais importantes, sendo aqueles com os quais a organização tem obrigação moral, enquanto os stakeholders com legitimidade derivativa são menos importantes e são aqueles com capacidade de afetar a relação entre a organização e seus stakeholders com legitimidade normativa. A questão do atendimento de interesses é discutida quando o autor afirma que os stakeholders com legitimidade normativa devem ser tratados conforme o princípio de fairness, ou seja, devem ser atendidos de forma justa, correspondente à sua contribuição para a empresa. 


\subsection{Contribuição para o modelo de priorização}

A fim de contribuir para o desenvolvimento de pesquisas que tratem do tema priorização, buscou-se destacar as contribuições que alguns dos estudos analisados podem oferecer ao modelo de priorização nas dimensões de percepção e atendimento. O Quadro 1 sintetiza as principais contribuições dos artigos, ou seja, variáveis que podem ser analisadas em futuros estudos de priorização.

Quadro 1 - Contribuição dos estudos de priorização

\begin{tabular}{|c|l|}
\hline Saliência & $\begin{array}{l}\text { A priorização do stakeholder pode ser entendida pela saliência, que é resul- } \\
\text { tante da combinação de três atributos: poder, legitimidade e urgência do } \\
\text { stakeholder (MITCHELL; AGLE; WOOD, 1997). }\end{array}$ \\
\hline Poder & $\begin{array}{l}\text { O poder do stakeholder afeta a percepção dos gestores quanto ao grau de } \\
\text { importância do stakeholder (FROOMAN, 2010; CHIGONA et al., 2010). }\end{array}$ \\
\hline Legitimidade & $\begin{array}{l}\text { As demandas dos stakeholders com legitimidade normativa devem ser prioriza- } \\
\text { das em relação aos stakeholders com legitimidade derivativa (PHILLIPS, 2003a). }\end{array}$ \\
\hline Influência & $\begin{array}{l}\text { A influência do stakeholder na organização afeta a percepção dos gestores } \\
\text { acerca do grau de importância do stakeholder (CHIGONA et al., 2010; PEDER- } \\
\text { SEN, 2011). }\end{array}$ \\
\hline $\begin{array}{c}\text { Estratégia da empresa } \\
\text { Contribuição do stake- } \\
\text { holder }\end{array}$ & $\begin{array}{l}\text { A percepção da importância do stakeholder é influenciada pela estratégia da } \\
\text { empresa em relação ao meio ambiente (HENRIQUES; SADORSKY, 1999; BUYS- } \\
\text { SE; VERBEKE, 2003). }\end{array}$ \\
\hline $\begin{array}{l}\text { O nível de atendimento pode considerar a contribuição do stakeholder para a } \\
\text { empresa (PHILLIPS, 2003a; CAMERON et al., 2011). }\end{array}$ \\
\hline $\begin{array}{c}\text { Função-objetivo da em- } \\
\text { presa }\end{array}$ & $\begin{array}{l}\text { Se a empresa adota a função-objetivo da teoria dos stakeholders, mesmo } \\
\text { que os acionistas não sejam atendidos, os demais stakeholders são atendidos } \\
\text { (BOAVENTURA et al., 2009). }\end{array}$ \\
\hline $\begin{array}{c}\text { Pressão do stakeholder } \\
\text { A percepção da importância do stakeholder, moderada pela pressão do stake- } \\
\text { holder para o atendimento de suas demandas relacionadas ao meio ambien- } \\
\text { Ne, influencia o atendimento dos interesses dos stakeholders (MURILLO-LU- } \\
\text { NARCES-AYERBE; RIVERA-TORRES, 2008; VAZQUEZ-BRUST et al., 2010). }\end{array}$ \\
\hline
\end{tabular}

Fonte: elaborado pelos autores.

Nos estudos analisados, é possível constatar que diferentes variáveis podem estar associadas à percepção de importância do stakeholder, como a saliência, o poder, a legitimidade, a influência do stakeholder e a estratégia da empresa, no caso, a estratégia ambiental. Outras variáveis estão associadas ao atendimento dos interesses dos stakeholders, como a contribuição do stakeholder e a função-objetivo da empresa. Alguns estudos contribuem ao enfatizar que a pressão que o stakeholder exerce na empresa, influenciada pela percepção da importância do stakeholder, pode afetar o nível de atendimento de seus interesses.

Com o estudo bibliográfico realizado nesta pesquisa, identificaram-se algumas variáveis que podem estar associadas tanto à percepção de importância do stakeholder quanto ao nível de atendimento do stakeholder. A partir dos resultados obtidos, é proposto um modelo apresentado na Figura 5. 


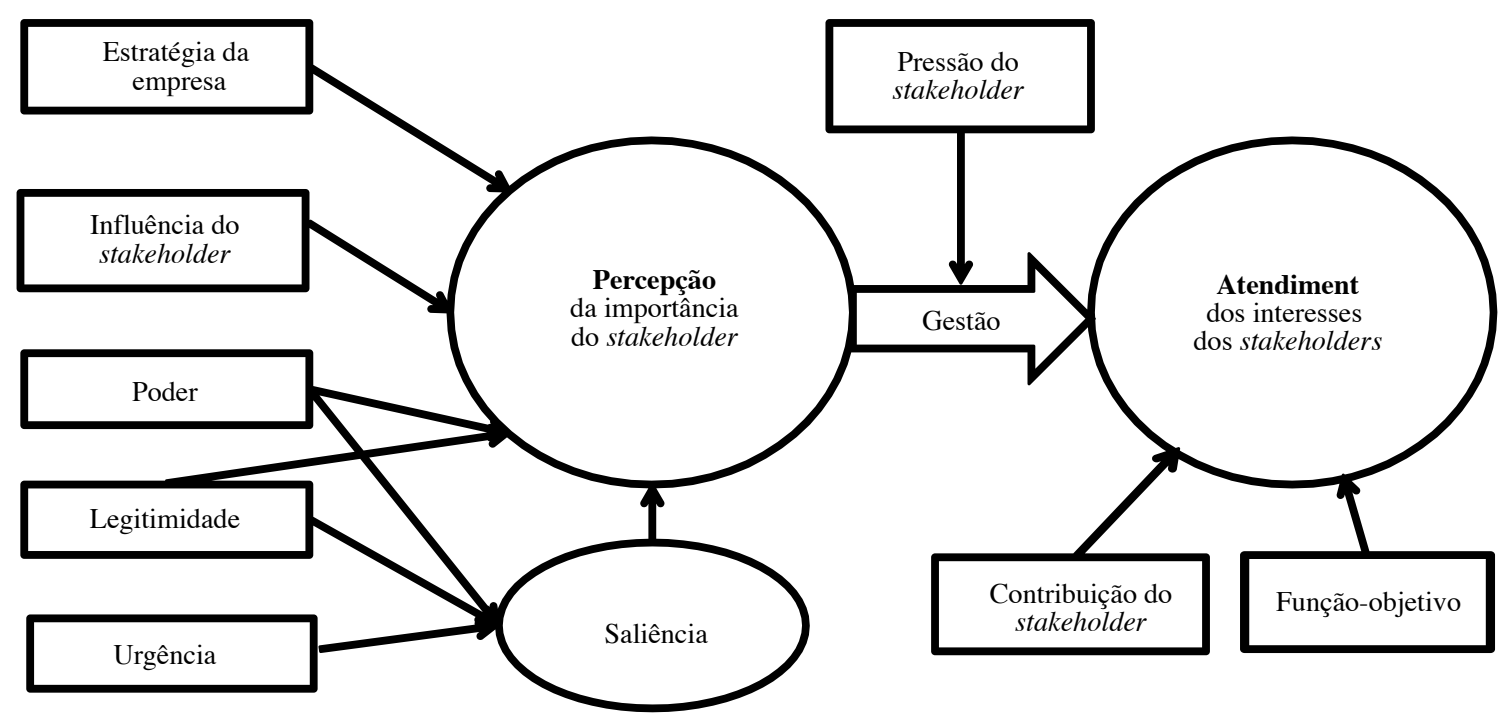

Figura 5 - Modelo de priorização de stakeholders obtido no estudo bibliográfico Fonte: elaborado pelos autores.

Na Figura 5, observa-se que os estudos de priorização contribuem com diferentes variáveis que podem ser mensuradas para a avaliação da priorização de stakeholders. A percepção de importância pode ser considerada um construto formado pela saliência, que, por sua vez, é um construto formado por poder, legitimidade e urgência. Estudos também defendem a relação entre poder e percepção e entre legitimidade e percepção, independentemente da saliência. A variável urgência estaria ligada apenas à saliência e caracteriza-se como um atributo temporário relacionado ao claim do stakeholder. Além dessas variáveis, a influência do stakeholder e a estratégia da empresa em relação ao meio ambiente também são consideradas em estudos da percepção de importância dos stakeholders.

$\mathrm{O}$ atendimento aos interesses dos stakeholders é considerado a partir de duas variáveis: a contribuição do stakeholder para a empresa, de modo que o atendimento em função da contribuição caracterizaria o princípio de fairness; e a função-objetivo da empresa, avaliando se está orientada para shareholders ou para stakeholders.

Por fim, relacionando a percepção ao atendimento, está a gestão de stakeholders, que pode ser afetada pela pressão dos stakeholders para o atendimento de suas demandas. Ressalta-se que é necessário um aprofundamento do modelo em futuros estudos de priorização para analisar se haveria de fato uma relação entre as variáveis e qual o tipo e a direção desta relação.

\section{CONSIDERAÇÕES FINAIS}

Esta pesquisa teve como objetivo geral analisar os conceitos de priorização de stakeholders adotados na literatura, propondo os seguintes objetivos específicos: descrever os conceitos, critérios ou modelos teóricos de priorização de stakeholders adotados na literatura; e analisar as contribuições das pesquisas sobre priorização de stakeholders.

Quanto ao primeiro objetivo específico - descrever os conceitos, critérios ou modelos teóricos de priorização de stakeholders -, confirmou-se o modelo de Boaventura et al. (2013) de que a priorização é baseada em dois conceitos: a percepção da importância dos stakeholders e o nível de atendimento dos interesses ou das demandas dos stakeholders. Na pesquisa realizada, observou-se que a maioria dos artigos (87\%) adota o conceito relacionado à percepção. Observaram-se diferentes critérios e modelos de hierarquização de stakeholders. Porém, constatou-se 
que o modelo de saliência de stakeholders é o mais adotado, correspondendo a $65 \%$ dos artigos da amostra. Os resultados apontam, assim, para uma ampla aceitação do modelo de Mitchell, Agle e Wood (1997) para avaliar a priorização de stakeholders.

Quanto ao segundo objetivo específico - analisar as contribuições das pesquisas sobre priorização de stakeholders -, observaram-se diferentes variáveis relacionadas à percepção de importância do stakeholder, como a saliência, o poder, a legitimidade, a influência do stakeholder e a estratégia da empresa, no caso, a estratégia ambiental. Outras variáveis estão associadas ao atendimento dos interesses dos stakeholders, como a contribuição do stakeholder e a função-objetivo da empresa. Alguns estudos contribuem ao enfatizar que a pressão do stakeholder afeta a gestão de stakeholders da organização, ou seja, a pressão que o stakeholder exerce, influenciada pela percepção da importância do stakeholder, pode afetar o nível de atendimento de seus interesses.

Ao analisar os resultados, observa-se que a priorização de stakeholders se relaciona com variáveis de difícil mensuração, o que pode levar a uma tendência no desenvolvimento de estudos sobre percepção, que pode ser avaliada por meio de survey com gestores ou estudo de caso. A obtenção de dados empíricos para avaliar o grau de atendimento dos stakeholders torna-se um desafio devido à complexidade de mensurar e comparar o nível de atendimento de diferentes interesses dos stakeholders. Por exemplo, o stakeholder funcionário pode ter como interesse mais oportunidades de carreira; e o stakeholder fornecedor pode ter como interesse desenvolver uma relação contínua e de confiança. Medir e comparar o atendimento de diferentes tipos de interesses é de difícil operacionalização. A partir da análise dos artigos, também se pode observar a amplitude do uso do termo stakeholder, uma vez que este é usado em diferentes estudos que não aprofundam a teoria. Ademais, muitos estudos investigam a priorização de issues, principalmente relacionada ao meio ambiente e não à priorização de stakeholders.

Para a evolução dos estudos sobre priorização de stakeholders, sugere-se uma agenda de pesquisa com três questões que podem ser aprofundadas em estudos futuros. A primeira questão de pesquisa refere-se a quais características dos stakeholders estão mais associadas à priorização. Este estudo identificou algumas características dos stakeholders, como a sua saliência (MITCHELL; AGLE; WOOD, 1997), o atributo de poder (FROOMAN, 2010; CHIGONA et al., 2010) e a legitimidade de suas demandas (PHILLIPS, 2003a). A segunda questão de pesquisa refere-se a quais comportamentos dos stakeholders estão mais associados à priorização. Este estudo mapeou alguns comportamentos dos stakeholders, tais como a influência do stakeholder na organização (CHIGONA et al., 2010; PEDERSEN, 2011), a pressão do stakeholder (MURILLO-LUNA; GARCES-AYERBE; RIVERA-TORRES, 2008; VAZQUEZ-BRUST et al., 2010) e a contribuição do stakeholder para a empresa (PHILLIPS, 2003a; CAMERON et al., 2011). A terceira questão de pesquisa refere-se a quais características da empresa estão mais associadas à priorização dos stakeholders. Este estudo identificou algumas características da empresa, como a sua função-objetivo (BOAVENTURA et al., 2009) e a sua estratégia em relação ao meio ambiente (HENRIQUES; SADORSKY, 1999; BUYSSE; VERBEKE, 2003).

Ressalta-se que este estudo apresenta limitações: a seleção de artigos em uma única base de dados, a ISI Web of Knowledge, bem como os critérios de palavras de busca e o período de pesquisa. Ademais, a análise qualitativa dos artigos para identificação das variáveis está sujeita a vieses dos pesquisadores.

A contribuição da pesquisa consiste em apresentar uma evolução do modelo de priorização de stakeholders baseado nos conceitos de percepção da importância dos stakeholders e no atendimento dos interesses dos stakeholders. O modelo proposto pode contribuir para que estudos posteriores aprofundem as relações entre as variáveis levantadas e explorem a agenda de pesquisa proposta para análise da priorização de stakeholders. 


\section{REFERÊNCIAS}

ADAMS, Renée B.; LICHT, Amir N.; SAGIV, Lilach. Shareholders and stakeholders: How do directors decide? Strategic Management Journal, v. 32, n. 12, p. 1331-1355, 2011.

AGUIAR, Fernando; BECKER, Alice; MILLER, Luis. Whose impartiality? An experimental study of veiled stakeholders, involved spectators and detached observers. Economics and Philosophy, v. 29, n. 2, p. 155-174, 2013.

ARAÚJO, Carlos A. Bibliometria: evolução histórica e questões atuais. Em Questão, v. 12, n. 1, p. 11-32, 2006.

BARDIN, Laurence. Análise de conteúdo. Lisboa: Edições 70, 1977.

BOAVENTURA, João Maurício Gama et al. Teoria dos stakeholders e teoria da firma: um estudo sobre a hierarquização das funçõesobjetivo em empresas brasileiras. Revista Brasileira de Gestão de Negócios, v. 11, n. 32, p. 289-307, 2009.

BOAVENTURA, João Maurício Gama et al. Limites da Teoria dos Stakeholders: a Questão da Dominância. In: ENCONTRO DA ANPAD, 37., 2013, Rio de Janeiro. Anais... Rio de Janeiro: EnANPAD, 2013.

BUYSSE, Kristel; VERBEKE, Alain. Proactive environmental strategies: a stakeholder management perspective. Strategic Management Journal, v. 24, n. 5, p. 453-470, 2003.

CAMERON, Bruce G. et al. Strategic decisions in complex stakeholder environments: a theory of generalized exchange. Engineering Management Journal, v. 23, n. 3, p. 37-45, 2011.

CARDOSO, André Coimbra Félix et al. O processo de internacionalização e os aspectos socioambientais: o caso Embraer. Revista de Administração da UFSM, v. 1, n. 1, p. 57-70, 2008.
CHIGONA, Wallace et al. Investigating the impact of stakeholder management on the implementation of a public access project: the case of Smart Cape. South African Journal of Business Management, v. 41, n. 2, p. 39-49, 2010.

CLARKSON, Max E. A stakeholder framework for analyzing and evaluating corporate social performance. Academy of Management Review, v. 20, n. 1, p. 92-117, 1995.

DE BUSSY, Nigel M.; SUPRAWAN, Lokweetpun. Most valuable stakeholders: The impact of employee orientation on corporate financial performance. Public Relations Review, v. 38, n. 2, p. 280-287, 2012.

DONALDSON, Thomas; PRESTON, Lee E. The stakeholder theory of the corporation: Concepts, evidence, and implications. Academy of Management Review, v. 20, n. 1, p. 65-91, 1995.

DRIESSEN, Paul H.; HILLEBRAND, Bas. Integrating multiple stakeholder issues in new product development: An exploration. Journal of Product Innovation Management, v. 30, n. 2, p. 364-379, 2013.

ETZIONI, Amitai. Modern organizations. Englewood Cliffs: Prentice-Hall, 1964.

EVAN, William M.; FREEMAN, R. Edward. A stakeholder theory of the modern corporation: Katian capitalism. In: DONALDSON, T.; WERHANE, P. H. (Eds.). Ethical issues in business. Englewood Cliffs: Prentice-Hall, 1993. p.166-171.

FREEMAN, R. Edward et al. Stakeholder theory: the state of the art. New York: Cambridge University Press, 2010.

FREEMAN, R. Edward. Divergent stakeholder theory. Academy of Management Review, $v$. 24, n. 2, p. 233-236, 1999.

FREEMAN, R. Edward. Strategic management: a stakeholder approach. Boston: Pitman, 1984. 
FROOMAN, Jeff. The issue network: reshaping the stakeholder model. Canadian Journal of Administrative Sciences/Revue Canadienne des Sciences de l'Administration, v. 27, n. 2, p. 161-173, 2010.

GIL, Antonio Carlos. Métodos e técnicas de pesquisa social. 5. ed. São Paulo: Atlas, 2008.

GIOIA, Dennis A. Practicability, paradigms, and problems in stakeholder theorizing. Academy of Management Review, v. 24, n. 2, p. 228232, 1999.

GUILD, Wendy L. Relative importance of stakeholders: analysing speech acts in a layoff. Journal of Organizational Behavior, v. 23, n. 7, p. 837-852, 2002.

HAIR JR., Joseph F. et al. Fundamentos de métodos de pesquisa em administração. Porto Alegre: Bookman, 2005.

HENRIQUES, Irene; SADORSKY, Perry. The relationship between environmental commitment and managerial perceptions of stakeholder importance. Academy of Management Journal, v. 42, n. 1, p. 87-99, 1999.

JEPSEN, Anna Lund; ESKEROD, Pernille. Stakeholder analysis in projects: Challenges in using current guidelines in the real world. International Journal of Project Management, v. 27, n. 4, p. 335-343, 2009.

KNOX, Simon; MAKLAN, Stan; FRENCH, Paul. Corporate social responsibility: Exploring stakeholder relationships and programme reporting across leading FTSE companies. Journal of Business Ethics, v. 61, n. 1, p. 7-28, 2005.

MACHADO-DA-SILVA, Clóvis L.; AMBONI, Nério; CUNHA, Vera Carneiro. Produção Acadêmica em Administração Pública: Período 1983-88. In: ENCONTRO ANUAL DA ANPAD, 13., 1989, São Paulo. Anais... São Paulo: ANPAD, 1989.

MARCOUX, Alexei M. Balancing Act. In:
DESJARDINS, J. R.; MCCALL, J. J. Contemporary Issues in Business Ethics. 4. ed. Belmont, Califf: Wadsworth, 2000. p. 92-100.

MITCHELL, Ronald K.; AGLE, Bradley R.; WOOD, Donna J. Toward a theory of stakeholder identification and salience: Defining the principle of who and what really counts. Academy of Management Review, v. 22, n. 4, p. 853-886, 1997.

MORAIS NETO, Siqueira; PEREIRA, Maurício Fernandes; MACCARI, Emerson Antonio. Classificando ações de sustentabilidade: uma análise de conteúdo de entrevistas de líderes. Revista de Administração da UFSM, v. 5, n. 1, p. 110-125, 2012.

MURILLO-LUNA, Josefina L.; GARCÉS-AYERBE, Concepción; RIVERA-TORRES, Pilar. Why do patterns of environmental response differ? A stakeholders' pressure approach. Strategic Management Journal, v. 29 , n. 11, p. $1225-$ 1240, 2008.

NEUMANN, Bruce R.; ROBERTS, Michael L.; CAUVIN, Eric. Stakeholder value disclosures: anchoring on primacy and importance of financial and nonfinancial performance measures. Review of Managerial Science, v. 5, n. 2-3, p. 195-212, 2011

NEVILLE, Benjamin A.; BELL, Simon J.; WHITWELL, Gregory J. Stakeholder salience revisited: Refining, redefining, and refueling an underdeveloped conceptual tool. Journal of Business Ethics, v. 102, n. 3, p. 357-378, 2011.

PEDERSEN, Esben Rahbek Gjerdrum. All animals are equal, but...: management perceptions of stakeholder relationships and societal responsibilities in multinational corporations. Business Ethics: A European Review, v. 20, n. 2, p. 177-191, 2011.

PHILLIPS, Robert. Stakeholder legitimacy. Business Ethics Quarterly, v. 13, n. 1, p. 25-41, 2003a.

Stakeholder theory and organizational 
ethics. Berrett-Koehler Publishers: San Francisco, 2003b.

PHILLIPS, Robert; FREEMAN, R. Edward; WICKS, Andrew C. What stakeholder theory is not. Business Ethics Quarterly, v. 13, p. 479502, 2003.

SEGUI-ALCARAZ, Antoni. Development of a Classification of Spanish Credit Institutions Based on the Concept of Stakeholder. Engineering Economics, v. 23, n. 5, p. 442-451, 2012.

STERNBERG, Elaine. Just Business. New York: Oxford University Press, 2000.

SUCHMAN, Mark C. Managing legitimacy: Strategic and institutional approaches. Academy of Management Review, v. 20, n. 3, p. 571-610, 1995.

TASHMAN, Pete; RAELIN, Jonathan. Who and What Really Matters to the Firm. Business Ethics Quarterly, v. 23, n. 4, p. 591-616, 2013.

TEIXEIRA, Maria Gracinda Carvalho; MORAES, Ivy Bertão de. O diálogo com stakeholders na teoria e na prática: análise da relação de uma empresa pública do setor industrial com seus stakeholders, para a construção de uma política de responsabilidade social. Revista de Administração da UFSM, v. 6, p. 211-228, 2013.

VAZQUEZ-BRUST, Diego Alfonso et al. Stakeholders pressures and strategic prioritisation: An empirical analysis of environmental responses in Argentinean firms. Journal of Business Ethics, v. 91, n. 2, p. 171-192, 2010.

WOOD, Donna J. Corporate Social Performance Revisited. Academy of Management Review, v. 16, n. 4, p. 691-718, 1991. 